\title{
Change blindness and the primacy of object appearance
}

\author{
GEOFF G. COLE and SIMON P. LIVERSEDGE \\ University of Durham, Durham, England
}

\begin{abstract}
A large body of work suggests that the visual system is particularly sensitive to the appearance of new objects. This is based partly on evidence from visual search studies showing that onsets capture attention whereas many other types of visual event do not. Recently, however, the notion that object onset has a special status in visual attention has been challenged. For instance, an object that looms toward an observer has also been shown to capture attention. In two experiments, we investigated whether onset receives processing priority over looming. Observers performed a change detection task in which one of the display objects either loomed or receded, or a new object appeared. Results showed that looming objects were more resistant to change blindness than receding objects. Crucially, however, the appearance of a new object was less susceptible to change blindness than both looming and receding. We argue that the visual system is particularly sensitive to object onsets.
\end{abstract}

A critical feature of the mammalian visual system is the rapid orientation of covert attention toward ecologically important events. The allocation of covert attention precedes saccadic orienting that allows direct fixation of the event. Indeed, it seems reasonable to assume that Darwinian selection has ensured that potentially threatening stimuli, such as the approach of a predator, receive visual processing priority. It is unsurprising, therefore, that researchers have long been interested in the type of stimulus events that accrue such priority. Potential candidates include stimuli that suddenly move, appear, or loom toward the observer.

Experimental psychologists have typically assessed the issue of processing priority with a variation of a standard visual search task in which an irrelevant stimulus property (e.g., movement) happens to occur at the location of one of the search items. The item exhibiting the property, referred to as a singleton, is irrelevant in the sense that it is not predictive of the target location since the target is no more likely to occur at the singleton location than at any of the other items. Thus, for a display size of seven, there is a one in seven chance that the target will coincide with the singleton. If a processing benefit is observed when the target and singleton do happen to coincide, that stimulus may be said to have accrued processing priority relative to the nonsingleton items. For example, in the onset singleton task (Yantis \& Jonides, 1984; see Figure 1A),

This work was supported by U.K. Economic and Social Research Council Grant RES-000-22-0888 to the first author and U.K. Biotechnology and Biological Sciences Research Council Grant 12/S19168 to the second author. We thank Steve Franconeri, Richard Abrams, and an anonymous reviewer for their assistance in improving the original manuscript. Correspondence should be addressed to G. G. Cole, Department of Psychology, Science Park, University of Durham, South Road, Durham DH1 3LE, England (e-mail: g.g.cole@durham.ac.uk). participants search for a target letter that appears among distractors. In a display of, say, seven search items, one of these items appears later than the others and thus constitutes the onset singleton. Additionally, unlike the onset singleton, the remaining six nonsingleton items are created by the transformation of figure $8 \mathrm{~s}$ that are present before the search display appears. Results typically show that when the target happens to be the new item (i.e., the onset singleton), reaction time (RT) is relatively short in comparison with when the target is generated from one of the (old) figure 8s (e.g., Gellatly, Cole, \& Blurton, 1999; Yantis \& Jonides, 1984).

Initial results suggested that onset singletons were the only task-irrelevant, unique feature that could capture attention. For instance, Jonides and Yantis (1988) investigated whether a luminance singleton could also elicit capture (see Figure 1B). Their results showed that when a target element coincided with a luminance singleton, RT was not reduced. In another experiment, Jonides and Yantis showed that the same was also true of color singletons. Yantis and Egeth (1999) even showed that "rare" color singletons - that is, singletons that only appear occasionally - also failed to capture attention. Furthermore, Hillstrom and Yantis (1994) have shown that motion singletons also fail to capture attention.

The fact that onset singletons consistently showed attentional capture, whereas other types of singletons did not, led to the suggestion that the visual system is particularly sensitive to new objects (Yantis, 1993). However, more recent findings have cast doubt on the new-object priority hypothesis. For instance, Turatto and Galfano (2001) reported an experiment in which observers were required to detect a single target letter among seven distractors. Each item was presented inside a circle, one of which was a color singleton. Thus, for example, one green circle occurred among seven red circles (or vice versa). 


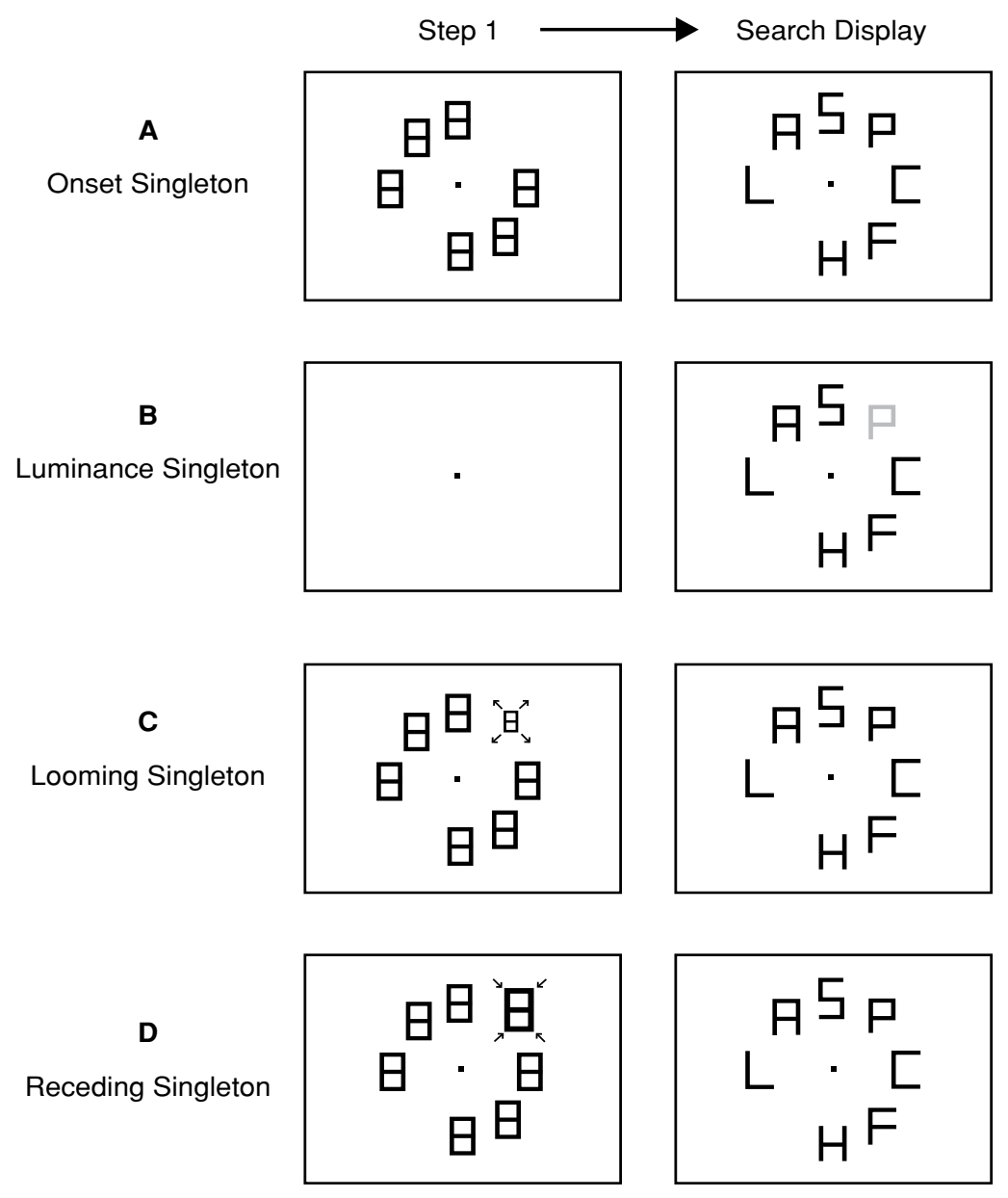

Figure 1. The singleton paradigm. In each of the four panels, one of the items in the display (i.e., the singleton) is unique with respect to a particular attribute. Occasionally the target element and singleton coincide. Typically $500 \mathrm{msec}$ elapse between the onset of the first step and the search display. No placeholder occurs with luminance singletons.

Unlike in previous color singleton experiments, RT facilitation occurred when the target letter appeared inside the unique color. Turatto and Galfano's crucial variation appears to have been that display set size was not manipulated. In a follow-up study, Turatto, Galfano, Gardini, and Mascetti (2004) demonstrated that this color singleton effect was not apparent when set size functions were analyzed. Johnson, Hutchison, and Neill (2001) have also provided evidence that color singletons can capture attention, while Franconeri and Simons (2003) and Abrams and Christ (2003) have shown how certain types of motion can do so. Finally, Kentridge, Cole, and Heywood (2004) reported that a singleton defined by local chromatic contrast attracted attention.

These findings have been taken as evidence that object onset is no more effective in marshalling attention than are other stimulus events. However, although these studies clearly demonstrate that other events do receive preferential processing, they do not reveal anything about the relative processing efficacy of such events in comparison with onset. In effect, the challenge to the special status of object onset has relied on the comparison of results from different studies or experiments. We believe that a more compelling demonstration of the relative efficacy of object onset in relation to other stimulus characteristics would be one in which different stimulus events are pitted against each other within the same experiments or even within the same trials. Indeed, since object onsets are now known not to be unique in capturing attention, new emphasis should be placed on determining which visual events have primacy relative to others.

Recently, Cole, Kentridge, and Heywood (2004) and Cole, Kentridge, Gellatly, and Heywood (2003) adopted this approach with the use of the change blindness paradigm. Change blindness refers to the phenomenon whereby observers often fail to notice a change to a display if the change coincides with other visual transients (e.g., Simons \& Rensink, 2005). One method for induc- 
ing change blindness is to briefly present a single uniformly blank image between the original scene and the subsequent scene containing the change. When the image reappears, participants may be required to indicate what changed or where the change occurred. This one-shot procedure contrasts with the more common continual alternation method whereby the two scenes are repeatedly presented alternately (with the blank image in between each) until the observer detects the change. The one-shot method essentially provides detection percentage correct data, whereas the continual alternation method provides RT data. Using the one-shot technique, Cole et al. (2004) compared the relative detectability of four different object attributes: appearance, disappearance, color change, and luminance change. Importantly, the low-level attributes of all the different stimulus properties were equated so that discriminability was the same for each. The results showed that object onset was least susceptible to change blindness. Furthermore, this effect held only for the onset of an object representation; onsets that did not create a new object did not accrue preferential processing.

Cole et al. (2004) concluded that the new onsets accrued processing priority over color change, luminance change, and object offset. Indeed, it is our contention that the degree to which different stimulus attributes render that stimulus more or less susceptible to change blindness provides an index of prioritization. This rationale is analogous to that of many other experimental paradigms in which relative primacy is used to assess and make inferences about cognitive processes such as attention. For instance, facilitated performance at cued locations in the precuing paradigm is usually taken as evidence that attention was located at the cued position (e.g., Posner \& Cohen, 1984). This conclusion is based solely on the observation that RTs are shorter relative to those at uncued locations. Likewise, many demonstrations of object-based attention (e.g., Duncan, 1984) are also based on the fact that performance is increased for the discrimination or detection of stimuli located within one single object relative to stimuli distributed across more than one object. The same rationale also applies to visual search experiments in which asymmetries in performance provide an index of primacy.

The rationale for employing the change detection paradigm does not, however, permit any conclusions regarding automatic attentional capture. As with the precuing paradigm, object-based attention studies, and many visual search studies, the technique enables one to infer only relative priority. Indeed, Yantis and Egeth (1999) have shown that many stimulus properties (e.g., color) that are highly salient, in the sense that they can be easily detected when observers are set to search for the property, fail to capture attention when they are irrelevant to the observer's task. Thus, if a phenomenally salient stimulus does not necessarily capture attention in a bottom-up fashion, then a change to a display in the change detection paradigm cannot in any way be said to do so.

The aim of the present research was to assess the new object priority hypothesis by comparing the degree to which a new object is resistant to change blindness in comparison with an object that looms toward the observer. Onset was compared with looming on the basis of a recent report that looming also captures attention. Franconeri and Simons (2003) employed a variation of the singleton paradigm in which one of the items grew in size. This induced the illusion of the item's looming toward the observer (see Figure 1C). Targets that coincided with the looming element accrued an RT benefit relative to targets that coincided with one of the nonlooming elements. Furthermore, this capture effect was not observed for stimuli that receded from the observer (see Figure 1D). Abrams and Christ (2005) have shown, however, that both looming and receding stimuli capture attention when simulated with stereo depth cues. They argued that it is actually motion onset that attracts attention (but see Franconeri \& Simons, 2005 , for a reply). It is apparent, therefore, that new objects are not unique in their ability to capture attention. However, as noted above, the discovery that other stimulus properties are able to capture attention does not refute the hypothesis that object onset has a special status and may still be the most effective stimulus property with respect to processing priority. In the present report, we compared change detection performance for stimuli that (1) appeared, (2) became larger, or (3) became smaller. Hence we induced the illusion of depth shift via size change. For clarity of presentation we shall refer to our stimuli as looming and receding. However, any differences we might observe in prioritization for each could be argued to be driven by the detection of size change rather than the detection of a shift in depth. Indeed, it is quite possible that size change might be the cue that the visual system uses to register looming and receding.

\section{EXPERIMENT 1}

The aim of Experiment 1 was to assess whether the basic effect whereby looming stimuli accrue enhanced processing relative to receding stimuli (Franconeri \& Simons, 2003) could be obtained using the change detection paradigm. Clearly, if the method is not sufficiently sensitive to demonstrate the looming/receding difference, then it would be unlikely to reveal any processing advantage for object onset when pitted against looming. Observers were given a one-shot change detection task in which one of the display objects either increased or decreased in size to induce the illusion of a shift either toward or away from the observer.

\section{Method}

Participants. Ten undergraduate psychology students took part and were paid $£ 5$.

Stimuli and Apparatus. On each trial of the experiment, from 11 to 14 squares of varying color and luminance were presented at random locations inside a virtual rectangle measuring $8.5^{\circ}$ in height and $10.6^{\circ}$ in width. The squares measured either $0.9^{\circ}$ or $1.3^{\circ}$ along each side. In other words, the display consisted of small and large squares. These were presented on a light purple background. The color coordinates of the background, measured in $L^{*} u^{*} v^{*}$ color space (using a Cambridge Research Systems ColorCAL colorimeter), were $0.286\left(u^{\prime}\right)$ and $0.284\left(v^{\prime}\right)$. The ratio of small to large squares 
was chosen pseudorandomly, with the restriction that at least 5 small or large squares would always appear. Thus any one trial was not dominated by either small or large squares. The squares would form two groups on either side of fixation with an approximately equal number appearing in each group. An example of the display is shown in Figure 2. The experiment was carried out in a single dimly lit room and was driven by a Pentium PC linked to an Eizo color monitor running at $60 \mathrm{~Hz}$. Participants were seated approximately $70 \mathrm{~cm}$ from the display and responded by pressing either the forward slash or backward slash on a standard keyboard.

Design and Procedure. A single factor (looming vs. receding) within-participants design was employed. Each trial began with the presentation of a fixation point for $1,000 \mathrm{msec}$ before the appearance of the first image for $1,200 \mathrm{msec}$. A blank frame of $100 \mathrm{msec}$ then followed before the onset of the second image for 1,200 msec. The blank frame then reappeared until the participant responded. For half the trials, one of the small squares became large when the display reappeared, whereas on the other half, one of the large squares became small. As with Franconeri and Simons's (2003) size change stimuli, this induced the perception of an object's having moved toward the observer or having moved farther away. Half of these changes occurred on the left-hand side of fixation and half on the right. Participants were asked to indicate whether they detected a change on either the right or the left side of fixation by pressing a right-hand or left-hand button. They were invited to guess if they did not detect any change. The beginning of a trial was initiated by the participant's response on the previous trial. Participants were instructed to maintain fixation for the entire duration of each trial and were told that accuracy, not speed, was important. They were not told what types of changes would occur, only that a single change would occur across the two images. For every trial on which an object loomed, the same pair of images would be repeated with the presentation order of the two images being reversed to create a receding trial. In other words, a looming object on one trial would become a receding object on another, and vice versa. This important control ensured that neither looming nor receding objects had an unfair advantage in detection

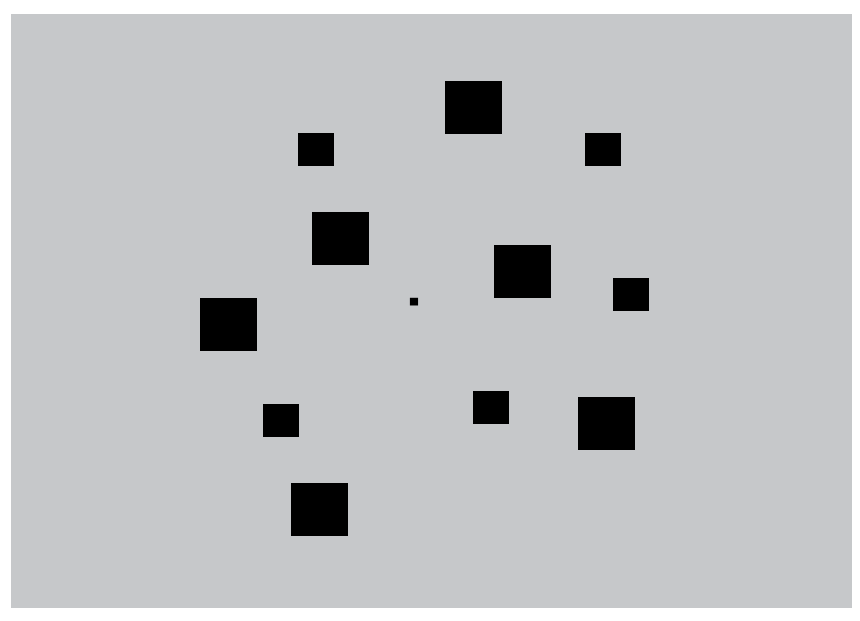

Figure 2. The type of display used in the present experiments. On each trial of the two experiments, the image would occur for $1,200 \mathrm{msec}$, offset for $100 \mathrm{msec}$, reappear for $1,200 \mathrm{msec}$, and then offset again. Participants were then required to indicate whether a change occurred on the left- or the right-hand side. When the image reappeared, one of the objects became larger or smaller (Experiment 1). This induced the illusion of movement either toward the observer or away from the observer (i.e., looming or receding). In Experiment 2, either an object became larger or a new object appeared. Note that the actual display consisted of differently colored objects against a light purple background. over the whole experiment. Thus, if, for example, a looming object had an advantage on one trial due to, say, its location nearer to fixation, this object would recede on another trial. Twenty practice trials were given, following a demonstration trial. One hundred twentyeight trials were then presented in the experiment. All trial types were presented randomly.

\section{Results and Discussion}

On average, participants detected a looming object on $66 \%$ of the trials and a receding object on $54 \%$ of the trials. Each observer's correct detection score for looming and receding was entered into a within-participants $t$ test. The difference in detection rates proved to be significant $[t(9)=2.8, p<.02]$. These data clearly show that an object that moves toward the observer is less susceptible to change blindness than is an object that moves farther away. This preferential processing for looming stimuli concurs with the findings reported by Franconeri and Simons (2003). We can conclude, therefore, that the change detection procedure employed previously by Cole et al. (2003; Cole et al., 2004) is sufficiently sensitive to reveal the looming/receding difference.

\section{EXPERIMENT 2}

The aim of Experiment 2 was to investigate, again using the change detection paradigm, the comparative effectiveness with which object onset and object looming are detected. Given that both looming and onset stimuli capture attention, in Experiment 2, we pitted object onset and object looming against each other within the same experimental item set, in order to determine their relative processing efficacies. In specific terms, we predicted that if the appearance of a new object has a special status in visual attention, a smaller change blindness effect should occur in the object onset condition than in the looming object condition.

\section{Method}

Participants. Ten undergraduate psychology students who did not take part in Experiment 1 were paid $£ 5$ to participate in Experiment 2.

Stimuli and Apparatus. For the looming condition, every aspect of the stimuli was identical to those reported in Experiment 1. The new object consisted of a square that measured $0.9^{\circ}$ along each side. In other words, the onset object was always a small square. As stated previously, when one compares the relative detectabilities of different stimulus properties in the change detection procedure, it is important to match the different stimuli for low-level characteristics. We therefore constructed the stimuli so that the size of the onset was the same as the increase in size of the looming object. Put another way, 576 pixels changed color at the same location in both onset and looming conditions: In the former condition, this created the onset of an object; in the latter condition, this created an increase in object size.

Design and Procedure. All aspects of these were as described for Experiment 1. Thus, observers were given a change detection task and were required to indicate whether the change occurred on the left or the right side of fixation. On half of the trials, a new object appeared; on the other half, an object loomed toward the observer.

\section{Results and Discussion}

Observers detected a looming object on $68 \%$ of the trials and a new object on $81 \%$. Each observer's correct detec- 
tion score for the new object and looming conditions was entered into a within-participants $t$ test. This difference was significant $[t(9)=7.8, p<.01]$. This result clearly shows that the appearance of a new object was more resistant to change blindness than an object that loomed. Importantly, our data represent the first demonstration within a single experiment that object onset is significantly more efficacious with respect to prioritization of visual processing than is object looming. This finding supports the claim that object onset has a special status in vision.

\section{GENERAL DISCUSSION}

In two experiments, we investigated the degree to which object onset, object looming, and object receding are susceptible to change blindness. Experiment 1 showed that an object that loomed toward the observer was more readily detected than an object that receded. This finding concurs with data reported by Franconeri and Simons (2003), who showed that looming stimuli gave rise to efficient visual search, whereas receding stimuli did not. Thus, Experiment 1 demonstrated that the change detection procedure produced a finding similar to that reported by Franconeri and Simons (2003). Experiment 2 then used this technique to assess whether the appearance of a new object would lead to facilitated visual processing in comparison with an object that loomed. The results showed this to be the case; object onset was more resistant to change blindness. We argue that this demonstrates visual processing priority for new objects over looming objects. Unlike previous studies, the present research assessed the new object priority hypothesis when onset was compared with another stimulus property within the same experimental item set.

These results support the notion that the visual system is particularly sensitive to the appearance of new objects, with object onset receiving attentional priority over both looming and receding. However, it is important to note that the findings may additionally implicate mechanisms of visual short-term memory as well as attention. It is not entirely clear to what extent the change detection paradigm engages primarily attention or memory processes. Since the degree of change blindness can be modulated by attentional factors (Rensink, O'Regan, \& Clark, 1997), the phenomenon must be, at least to a certain extent, an attentional effect by definition. At the same time, the paradigm of comparing one image with a subsequent image clearly requires memory. Indeed, a visual short-term memory mechanism may help to explain new object prioritization. Despite poor retention of much information within visual short-term memory, change in the spatial configuration of objects is relatively well retained (Simons, 1996). In the present Experiment 2, the spatial layout of objects altered with the appearance of the new object. By contrast, no such alteration occurred when the old object changed size in the looming condition. Since new objects necessarily change the spatial layout of a scene, correct change detection would therefore be greater on new object trials. Given this, the present data may actually reflect priority for representations of changes in spatial configuration, with facilitated new object detection being one of the consequences. This account could potentially explain all reported demonstrations of new object priority (e.g., Yantis \& Jonides, 1984). Alternatively, spatial layout changes may be the cue that the system uses to register the appearance of new objects. Hence, rather than being epiphenomenal, new object priority would still have a special status in visual attention but would use spatial configuration as a cue.

Finally, although the present data demonstrate primacy for object onset, the results do not allow us to form conclusions about whether or not new onsets automatically capture attention. As we have stated in the introduction, our use of the change detection paradigm allows us only to assess which visual events have priority, rather than which events lead to capture per se. Clearly, there may well be a relationship between capture and the attenuation of change blindness, but attenuation does not necessarily demonstrate capture. We do, however, argue that since attentional capture and processing priority can be independent, the finding that new onsets may not necessarily capture attention (e.g., Franconeri, Hollingworth, \& Simons, 2005) does not refute the hypothesis that object onsets have a special status with respect to the prioritizing of visual events.

\section{REFERENCES}

Abrams, R. A., \& Christ, S. E. (2003). Motion onset captures attention. Psychological Science, 14, 427-432.

Abrams, R. A., \& Christ, S. E. (2005). The onset of receding motion captures attention: Comment on Franconeri and Simons (2003). Perception \& Psychophysics, 67, 219-223.

Cole, G. G., Kentridge, R. W., Gellatly, A. R. H., \& Heywood, C. A. (2003). Dectectability of onsets versus offsets in the change detection paradigm. Journal of Vision, 3, 22-31.

Cole, G. G., Kentridge, R. W., \& Heywood, C. A. (2004). Visual salience in the change detection paradigm: The special role of object onset. Journal of Experimental Psychology: Human Perception \& Performance, 30, 464-477.

DunCAN, J. (1984). Selective attention and the organization of visual information. Journal of Experimental Psychology: General, 113, 501517.

Franconeri, S. L., Hollingworth, A., \& Simons, D. J. (2005). Do new objects capture attention? Psychological Science, 16, 275-282.

Franconeri, S. L., \& Simons, D. J. (2003). Moving and looming stimuli capture attention. Perception \& Psychophysics, 65, 999-1010.

Franconeri, S. L., \& Simons, D. J. (2005). The dynamic events that capture visual attention: A reply to Abrams and Christ (2005). Perception \& Psychophysics, 67, 962-966.

Gellatly, A. [R. H.], Cole, G. [G.], \& Blurton, A. (1999). Do equiluminant object onsets capture visual attention? Journal of Experimental Psychology: Human Perception \& Performance, 25, 1609-1624.

Hillstrom, A. P., \& Yantis, S. (1994). Visual motion and attentional capture. Perception \& Psychophysics, 55, 399-411.

Johnson, J. D., Hutchison, K. A., \& Neill, W. T. (2001). Attentional capture by irrelevant color singletons. Journal of Experimental Psychology: Human Perception \& Performance, 27, 841-847.

Jonides, J., \& YANTIS, S. (1988). Uniqueness of abrupt visual onset in capturing attention. Perception \& Psychophysics, 43, 346-354.

Kentridge, R. W., Cole, G. G., \& Heywood, C. A. (2004). The primacy of chromatic edge processing in normal and cerebrally achromatopsic subjects. Progress in Brain Research, 144, 161-169.

Posner, M. I., \& Cohen, Y. (1984). Components of attention. In 
H. Bouma \& D. G. Bouwhuis (Eds.), Attention and performance X: Control of language processes (pp. 55-66). Hillsdale, NJ: Erlbaum.

Rensink, R. A., O'Regan, J. K., \& Clark, J. J. (1997). To see or not to see: The need for attention to perceive changes in scenes. Psychological Science, 8, 368-373.

Simons, D. J. (1996). In sight, out of mind: When object representations fail. Psychological Science, 7, 301-305.

Simons, D. J., \& Rensink, R. A. (2005). Change blindness: Past, present, and future. Trends in Cognitive Sciences, 9, 16-20.

Turatto, M., \& Galfano, G. (2001). Attentional capture by color without any relevant attentional set. Perception \& Psychophysics, 63, 286-297.

Turatto, M., Galfano, G., Gardini, S., \& Mascetti, G. G. (2004). Stimulus-driven attentional capture: An empirical comparison of display-size and distance methods. Quarterly Journal of Experimental Psychology, 57A, 297-324.

Yantis, S. (1993). Stimulus-driven attentional capture. Current Directions in Psychological Science, 2, 156-161.

YANTIS, S., \& EgeTH, H. E. (1999). On the distinction between visual salience and stimulus-driven attentional capture. Journal of Experimental Psychology: Human Perception \& Performance, 25, 661-676.

YANTIS, S., \& Jonides, J. (1984). Abrupt visual onsets and selective attention: Evidence from visual search. Journal of Experimental Psychology: Human Perception \& Performance, 10, 601-621.

(Manuscript received April 1, 2005;

revision accepted for publication December 19, 2005.) 